\title{
Modification of the Development Parameter for a Chemically Amplified Resist Simulator
}

\author{
Eun-Jung SEO,* Heung-Jin BAK, Sang-Kon KIm, Young-Soo SoHn and Hye-Keun OH \\ Department of Physics, Hanyang University, Ansan 425-795
}

(Received 11 February 2001)

\begin{abstract}
It is necessary to have more appropriate resist parameters in order for a lithography simulator to predict real photoresist profiles. These process parameters are usually obtained by flood exposure experiments without pattern masks. However, real processes are performed with pattern masks. Since the intensity on the wafer is different with and without a pattern, the development parameters must be modified in order to predict real processes. Especially, the development parameters, one example of the process parameters, are crucial to mimic real processes. It has been reported that the development parameters of a photoresist with or without underlying patterns are different. In this paper, we modified the flood exposure development parameters of a 248-nm chemically amplified resist (CAR) to get patterned development parameters and compared them with the simulation results. First, we obtained the development parameters by using a flood exposure experiment and applied them to our lithography simulator LUV. The simulated resist profiles were then compared to SEM microphotographs. Second, we modified the development parameters for the simulated resist profile to match the SEM photographs. We also determined the relationship between the changes of the parameters and the pattern profile. We could see the effect of the modification in different line widths and sidewall angle.
\end{abstract}

PACS numbers: 42.82.C

Keywords: Lithography, Chemically amplified resist, Development parameter

\section{INTRODUCTION}

A lithography simulation tool can mimic the real process only if the simulation parameters are given correctly. In most simulation tools, the parameters are modified to predict the resist profiles since all the parameters can not be obtained in real lithographic processes. The development parameters are usually obtained from flood exposure experiments without a pattern. However, the development parameters are different when a pattern exists. If the development parameters of flood exposure without modification were to be used, the simulated profiles were not consistent with the experimental results. In this research, we modified the development parameters of a $248-\mathrm{nm}$ chemically amplified resist (CAR) with mask patterns. We investigated the effects of each development parameter on the linewidth, the side wall angle, and the thickness of the profile. Among the development parameters, the change of the minimum development rate has little effect on the resist profile while the others cause significant variations in the profiles. As the maximum development rate and the threshold photo acid generator (PAG) concentration are increased, the linewidth is decreased and the side wall angle is in-

*E-mail: ppolpory@newton.hanyang.ac.kr creased. When dissolution selectivity is increased, the linewidth and the side wall angle are increased. These characteristics were taken into account to extract the optimum values of the development parameters for patterned resist profiles. The modified parameters were used to predict experimental profiles, and we obtained modified simulation results. The development parameters were then modified again to get the simulation profiles close to the experimental scanning electron microscope (SEM) images. The modification was repeated until the simulated profile matched the experimental results. We also compared the development parameters of flood and patterned exposures.

\section{MODIFICATION OF DEVELOPMENT PARAMETERS}

\section{Flood Exposure and Patterned Exposure}

Generally, exposure parameters [1], post-exposure bake (PEB) parameters [2], and development parameters are called the process parameters. Appropriate resist parameters are necessary for a lithography simulator to 


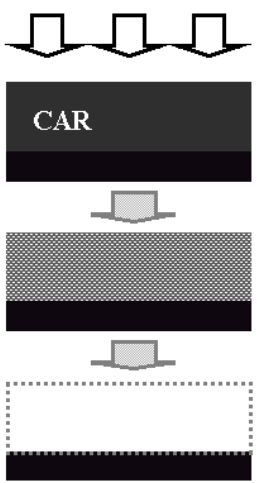

(a)

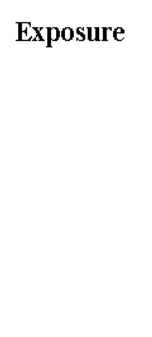

Development
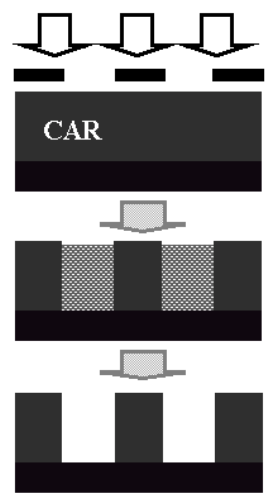

(b)

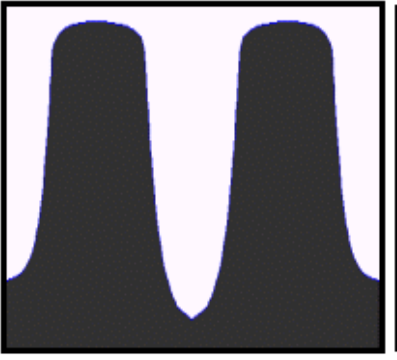

(a)

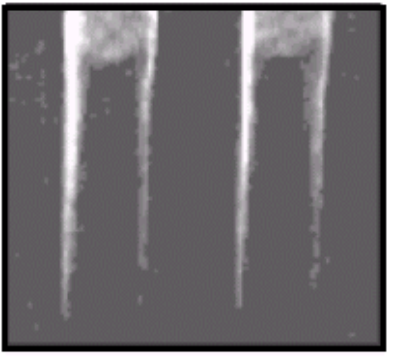

(b)
Fig. 2. Simulation profile applying the development parameters obtained by (a) flood exposure and (b) SEM image.

as

$$
\begin{aligned}
& R=R_{\max } \frac{(a+1)(1-m)^{n}}{a+(1-m)^{n}}+R_{\text {min }}, \\
& a=\frac{n+1}{n-1}\left(1-m_{t h}\right)^{n}
\end{aligned}
$$

is the maximum (fully exposed, $m=0$ ) dissolution rate, $R_{\text {min }}$ is the minimum (unexposed, $m=1$ ) dissolution rate, $n$ is the dissolution selectivity which controls the contrast of the photoresist, $m$ is the PAG concentration, and $m_{t h}$ is the threshold PAG. The development parameters can be obtained by using a development rate monitoring (DRM). [6] The resist was distributed on a wafer by spin coating and was then flood-exposed after a soft bake. The wafer was put into a developer, and the thickness change was measured with respect to time. The minimum development rate, $R_{\text {min }}$, and the maximum development rate, $R_{\max }$, were determined using the DRM. In order to calculate the minimum development rate, $R_{\text {min }}$, we obtained the thickness of a coated resist on a wafer that had not been exposed by using thickness measurement tool. After more than three hour, the wafer was removed from the developer, washed using deionized water, and dried with nitrogen; then, the thickness of the wafer was again measured. The minimum development rate was then calculated based on the thickness change in the film over the three hour period. In order to measure the maximum development rate, $R_{\max }$, a resist-coated wafer was exposed to a dose of $2000 \mathrm{~mJ} / \mathrm{cm}^{2}$, a sufficient dose to completely bleach the PAG in the resist film. This wafer was then developed and its development rate was monitored using the DRM. That development rate was set as $R_{\max }$. The other parameters, $m_{t h}$ and $n$, were determined by fitting $R(m)$ data with development rate models. $[7,8]$

\section{Simulation}

The Simulation was due using our in-house simulator LUV. When the usual parameters obtained by the flood
The simulation profile matching the SEM image can be posure dose but the development parameters. [5] There are many current development models. We used Mack's equation, one of the development rate equations, since the equation is generally used and provides a good fit to experimental data. The original Mack model is expressed 
Table 1. Exposure parameters and PEB parameters obtained by using flood exposure experiments

\begin{tabular}{c|c}
\hline \hline Dill parameter A $(1 / \mu \mathrm{m})$ & -0.010 \\
Dill parameter B $(1 / \mu \mathrm{m})$ & 0.3620 \\
Dill parameter B $\left(\mathrm{cm}^{2} / \mathrm{mJ}\right)$ & 0.0120 \\
$\mathrm{~K}_{\text {amp }}$ & 0.125889 \\
$\mathrm{~K}_{\text {loss }}$ & 0.009188 \\
\hline \hline
\end{tabular}

Table 2. Development parameters obtained by using flood exposure experiments

\begin{tabular}{c|c}
\hline \hline $\mathrm{R}_{\text {max }}(\mu \mathrm{m} / \mathrm{s})$ & 0.134 \\
$\mathrm{R}_{\text {min }}(\mu \mathrm{m} / \mathrm{s})$ & 0.004 \\
$\mathrm{~m}_{t h}$ & 0.3 \\
$\mathrm{n}$ & 5.2 \\
\hline \hline
\end{tabular}

experiments were used, the simulation result, shown in Fig. 2(a), did not agree with the SEM image. Figure 2(b) shows the SEM image of the commonly used 248 nm-CAR APEX-E for a $0.4-\mu \mathrm{m}$ line and space $(\mathrm{L} / \mathrm{S})$ pattern [9]. The illumination conditions were $\lambda=248$ $\mathrm{nm}, N A=0.42$, and $\sigma=0.5$, and the exposure dose was $6 \mathrm{~mJ} / \mathrm{cm}^{2}$. The PEB was done at $90{ }^{\circ} \mathrm{C}$ for $90 \mathrm{~s}$. Table 1 and Table 2 show the process parameters obtained by flood exposure for APEX-E.

We modified the development parameters to make the simulation profile agree with the SEM image. To extract the optimum development parameters, we modified each development parameter and observed the effect of each parameter on the linewidth and the sidewall angle. The results are shown in Figs. 3-6.

To know the effect of each development parameter on the simulated profile and to obtain suitable development parameters for simulation, we varied each development parameter a little bit around basic parameter obtained from the flood exposure. To check the effect of each development parameter, in the simulation, we used the varied value for one parameter and the values obtained from flood exposure experiments for the other values. Figure 3 shows the variation of linewidth and the sidewall angle of the pattern with $R_{\max }$. The value of $R_{\max }$ was varied from 0.1 to $0.6 \mu \mathrm{m} / \mathrm{s}$. When the value of $R_{\max }$ was
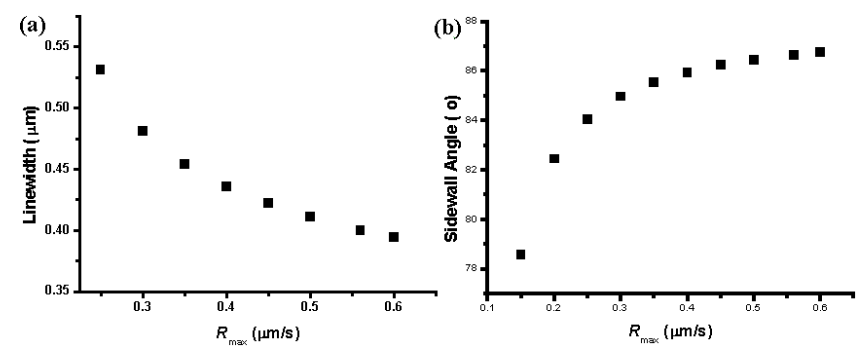

Fig. 3. Linewidth and sidewall angle vs. $R_{\max }$.

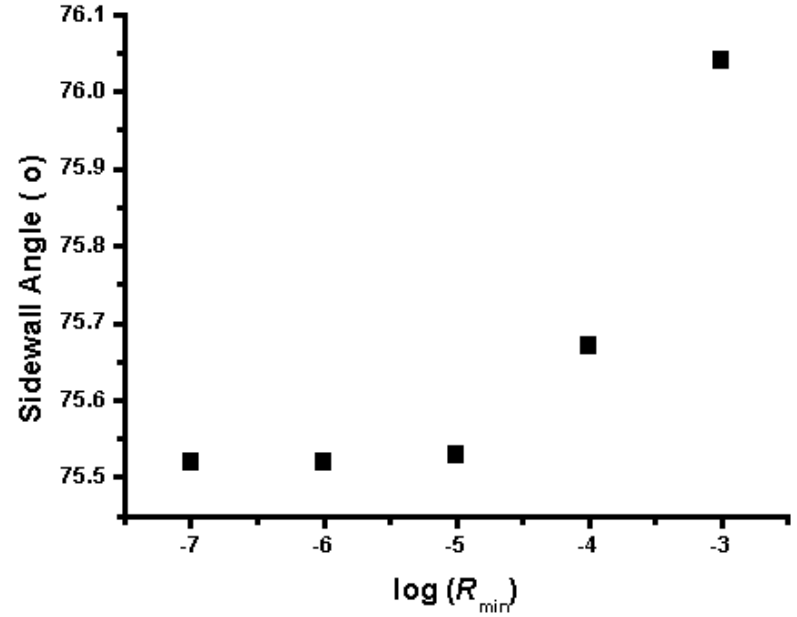

Fig. 4. Linewidth and sidewall angle vs. $R_{\min }$.

increased, the linewidth of the pattern profile decreased, and the sidewall angle increased. $R_{\min }$ is the dissolution rate of the unexposed area, so the variation of this value has nearly no effect on the linewidth and the sidewall angle of the resist profile, as seen in Fig. 4. The resist thickness varies significantly only when the $R_{\text {min }}$ value is very large. Figure 5 shows the variation of linewidth and sidewall angle of the resist profile when $m_{t h}$ varied. In Fig. 5, the value of $m_{t h}$ varies from 0.4 to 0.6 . When $m_{t h}$ was increased, the line width of the pattern profile decreased and the sidewall angle increased. The effect of $m_{t h}$ is similar to that of $R_{\max }$. The effect of the dissolution selectivity $n$ is shown in Fig. 6. The value of $n$ was varied from 3 to 5 . When $n$ was increased, the linewidth of the pattern profile increased, and the sidewall angle
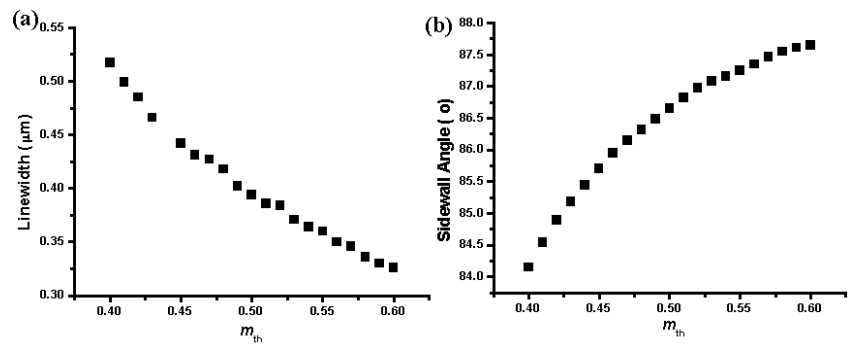

Fig. 5. Linewidth and sidewall angle vs. $m_{t h}$.
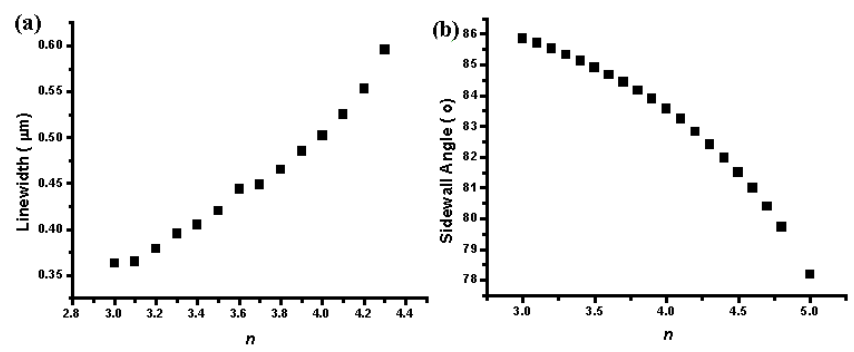

Fig. 6. Linewidth and sidewall angle vs. $n$. 
Table 3. Optimum development parameters for predicting the real result

\begin{tabular}{c|c}
\hline \hline $\mathrm{R}_{\max }(\mu \mathrm{m} / \mathrm{s})$ & 0.175 \\
$\mathrm{R}_{\min }(\mu \mathrm{m} / \mathrm{s})$ & 0.0004 \\
$\mathrm{~m}_{t h}$ & 0.35 \\
$\mathrm{n}$ & 4 \\
\hline \hline
\end{tabular}

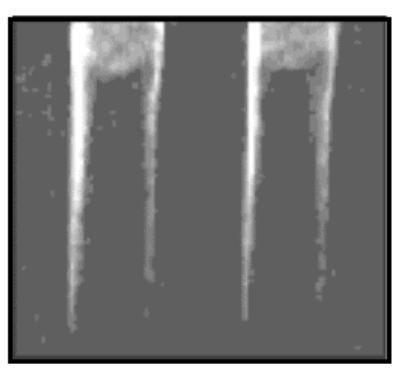

(a)

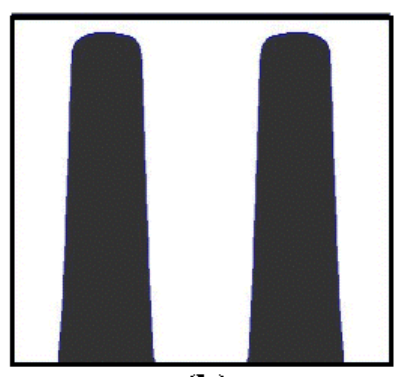

(b)
Fig. 7. (a) SEM image (same as Fig. 2(b)) and (b) simulation profile obtained by applying the modified optimum development parameters.

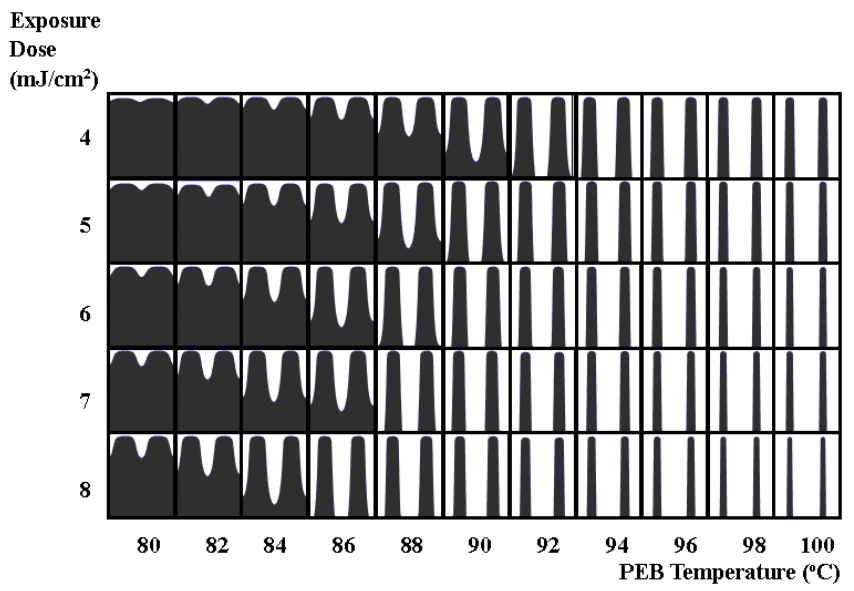

Fig. 8. Simulation resist profiles with respect to the exposure dose and PEB temperature.

decreased. When the number of $n$ is increased, the developed resistance of the resist is increased [10].

Each development parameter can affect the resist profile in a different way. Therefore, we performed a series of simulations until the simulation profile was close to the experimental result. We obtained the linewidth, the sidewall angle, and the thickness of the remaining resist, and we modified the parameters by considering the effects of each parameter. The resulting optimum parameters for a 400-nm L/S are shown in Table 3. The simulation profile with these optimum parameters is shown in Fig. 7(b).

Three optimum development parameters were applied to other process conditions, and we could obtain good simulation results. Figure 8 shows the simulated resist profiles with respect to exposure dose and PEB temper- ature. The exposure dose was $4 \sim 8 \mathrm{~mJ} / \mathrm{cm}^{2}$, and the PEB temperature was $80{ }^{\circ} \mathrm{C} \sim 100{ }^{\circ} \mathrm{C}$.

\section{CONCLUSION}

Generally, the parameters obtained from flood exposure experiments are used for lithography simulation to predict the real process result. However, in a real process, the exposure is performed with a patterned mask. Since on intensity difference exists between the flood exposure and the patterned exposure, if the parameters obtained from flood exposure experiments are used, the resulting simulation profile may be different from the real one. We modified the development parameters, instead of changing exposure dose value. The optimized development parameters were extracted by considering the effects of each parameter on the simulation profiles. We simulated 400-nm L/S patterns for a 248-nm CAR with the modified development parameters. We found that the change of $R_{\text {min }}$ had little effect on the profile while the other three parameters exerted a greater influence on the resist profile. The optimized development parameters were applied to other process conditions, and good simulation results could be obtained. We also found that larger values of the parameters were needed for smaller pattern sizes at the same pattern density.

\section{REFERENCES}

[1] Y. S. Sohn, E. M. Lee, M. G. Sung, Y. M. Lee, H. J. Bak, I. S. An and H. K. Oh, J. Korean Phys. Soc. 39, $152(2001)$

[2] H. K. Oh, Y. S. Sohn, M. G. Sung, Y. M. Lee, E. M. Lee and I. S. An, J. Korean Phys. Soc. 35, S734 (1999)

[3] S. Inoue, T. Fujisawa and K. Izuha, in Proc. SPIE (San Jose, June, 2000), p. 810.

[4] A. Sekiguchi, C. A. Mack, M. Isono and T. Matsuzawa, in Proc. SPIE (San Jose, Jan., 1999), p. 985.

[5] A. Sekiguchi, C. A. Mack, Y. Minami and T. Matsuzawa, in Proc. SPIE (San Diego/La Jolla, Feb., 1996), p. 49.

[6] S. D. Chowdhury, D. Alexander, M. Goldman, A. W. Kukas, N. Farrar, C. Takemoto, B. W. Smith and L. Karklin, in Proc. SPIE (San Diego/La Jolla, Feb., 1995), p. 659 .

[7] C. L. Henderson, P. C. Tsiartas, S. N. Pancholi, S. A. Chowdhury, K. D. Dombrowski, C. G. Willson and R. R. Dammel, in Proc. SPIE (San Jose, Feb., 1997), p. 805.

[8] S. H. Thornton and C. A. Mack, in Proc. SPIE (San Diego/La Jolla, Feb., 1996), p. 223.

[9] J. Fahey, W. K. Moreau, K. Welsh, S. Miura, M. Eib and G. Spinillo, in Proc. SPIE (San Jose, Feb., 1994), p. 422.

[10] T. Zhong, E. Gurer, J. Lewellen and E. Lee, in Proc. SPIE (San Jose, June, 2000), p. 793. 